\title{
Mean curvature of spacelike submanifolds in a Brinkmann spacetime*
}

\author{
Verónica L. Cánovas $^{a}$, Francisco J. Palomo $^{b}$ and Alfonso Romero ${ }^{c}$ \\ ${ }^{a}$ Departamento de Matemáticas, Universidad de Murcia, \\ Campus de Espinardo, 30100 Espinardo, Murcia, Spain, \\ E-mail: veronica.lopez10@um.es \\ ${ }^{b}$ Departamento de Matemática Aplicada, \\ Universidad de Málaga, \\ 29071 Málaga, Spain, \\ E-mail: fjpalomo@ctima.uma.es \\ ${ }^{c}$ Departamento de Geometría y Topología \\ Universidad de Granada \\ 18071 Granada, Spain, \\ E-mail: aromero@ugr.es
}

\begin{abstract}
Several geometric properties of complete spacelike submanifolds, with codimension at least two, in a Brinkmann spacetime are shown from natural assumptions involving the mean curvature vector field $\mathbf{H}$ of the spacelike submanifold. Especially, we get sufficient conditions that assure that a spacelike submanifold is contained in a leaf of the foliation of the Brinkmann spacetime defined by the orthogonal vectors to the parallel lightlike vector field. When this vector field is the gradient of a smooth function, a characterization of arbitrary codimension spacelike submanifolds contained in a leaf of this foliation is given. In the case of plane fronted wave spacetimes, relevant examples of Brinkmann spacetimes that generalize pp-waves spacetimes, several uniqueness results for codimension two spacelike submanifolds are obtained. In particular, it is proven that any compact codimension two spacelike submanifold with $\mathbf{H}=0$ in a plane fronted spacetime wave must be a (totally geodesic) front of wave.
\end{abstract}

Keywords: Brinkmann spacetime, plane fronted wave spacetime, pp-wave spacetime, gravitational plane wave spacetime, spacelike submanifold, front of wave, characteristic hypersurface, mean curvature vector field.

\footnotetext{
${ }^{* b}$ Corresponding author.

The first author has been partially supported by MINECO/FEDER project MTM2015-65430-P, Fundación Séneca project 19901/GERM/15 and research grant 19783/FPI/15 from Fundación Séneca, the second and the third authors by Spanish MINECO and ERDF project MTM2016-78807-C2-1-P and Andalusian and ERDF project A-FQM-494-UGR18.
} 


\section{Introduction}

Lorentzian manifolds $\left(\bar{M}^{n+2},\langle\rangle,\right)$ admitting a (globally defined) parallel and lightlike vector field $\xi$ were introduced in [7]. Such a Lorentzian manifold is time-orientable (as justified in Section 2) and then, endowed with the time orientation induced by $\xi$, it is an $(n+2)$-dimensional spacetime, called a Brinkmann spacetime. Each Brinkmann spacetime $\bar{M}^{n+2}$ admits a natural $(n+1)$-dimensional distribution, namely $D_{\xi}^{\perp}:=\{X \in \mathfrak{X}(\bar{M}):\langle X, \xi\rangle=0\}$. Indeed, $D_{\xi}^{\perp}$ is a foliation as shown in Section 2. Thus, a Brinkmann spacetime is foliated by the integral hypersurfaces (i.e., the leaves) of $D_{\xi}^{\perp}$. Each leaf of $D_{\xi}^{\perp}$ is called a characteristic hypersurface in $\bar{M}^{n+2}$. Note that such a hypersurface inherits a degenerate metric from the Lorentzian metric of $\bar{M}^{n+2}$. From a physical point of view, a leaf may be interpreted as describing a propagating gravitational front of wave.

The family of Brinkmann spacetimes includes plane fronted wave spacetimes [8] and then, also the so-called pp-wave spacetimes and gravitational plane wave spacetime [6], [11], which are exact solutions of the Einstein field equation modelling gravitational waves [22, p. 381]. The interest on this type of spacetimes comes from along time ago [18] and it has increased widely in recent years, due to the experimental detection of gravitational waves [1].

Our first aim is to investigate codimension greater than two spacelike submanifolds $\psi: \Sigma^{k} \rightarrow$ $\bar{M}^{n+2}$ in a Brinkmann spacetime $\bar{M}^{n+2}$ such that $\psi\left(\Sigma^{k}\right)$ is contained in a characteristic hypersurface. If this is the case, we will say that the submanifold factorizes through a characteristic hypersurface. We would like to point out that we consider here immersed spacelike submanifolds, thus the embedded ones, which have a more clear role in Relativity, remain as a relevant particular case, indeed, each codimension two embedded spacelike submanifold contained in a characteristic hypersurface may be seen as modelling the source of the gravitational front of wave.

The mean curvature vector field $\mathbf{H}$ of a $m$-codimensional spacelike submanifold codifies its extrinsic geometry. When $m=1$, i.e., for a spacelike hypersurface, $\mathbf{H}$ is described by the mean curvature function (relative to the time orientation of the spacetime). Spacelike hypersurfaces with zero mean curvature in a Brinkmann spacetime are been studied in [16] and, in the particular case when the Brinkmann spacetime is a pp-wave, in [23]. When $m \geq 2$, neither techniques nor reasonable physical motivation/interpretation come from the case $m=1$. Indeed, we first provide several conditions on $\mathbf{H}$ to assure the submanifold factorizes through a characteristic hypersurface. Then, in the philosophy of [3], [15] and [2], we try to obtain global uniqueness results as, for instance in the case the spacetime is a plane fronted wave.

The content of this paper is organized as follows. Section 2 is devoted to introduce some preliminaries and notation. The local description of the metric of a Brinkmann spacetime $\left(\bar{M}^{n+2},\langle\rangle,\right)$ with parallel lightlike vector field $\xi$ and foliation $D_{\xi}^{\perp}$ is exposed. Several direct properties for general $k$ dimensional spacelike submanifolds $\psi: \Sigma^{k} \rightarrow \bar{M}^{n+2}$ are then explained. In particular, formula 2.9. states a relationship between the mean curvature vector field $\mathbf{H}$ of $\Sigma^{k}$ and the tangential projection $\xi^{\top}$ of $\xi$ along $\psi$. As a consequence when $k=n$, if $\mathbf{H}$ is causal at any point and the spacelike sub- 
manifold $\Sigma^{n}$ of $\bar{M}^{n+2}$ is compact, then $\mathbf{H}$ must be lightlike everywhere and $\Sigma^{n}$ factorizes through a characteristic hypersurface.

For those Brinkmann spacetimes that satisfy $\xi^{b}=d U$ globally, $U \in C^{\infty}(\bar{M})$, Lemma 2.4 states that $\Delta(U \circ \psi)=k\langle\mathbf{H}, \xi\rangle$, where $\Delta$ denotes the Laplace operator on $\Sigma^{k}$. Thus, we get the following characterization for spacelike submanifolds through a characteristic hypersurface, Corollary 2.5,

A spacelike submanifold $\psi: \Sigma^{k} \rightarrow \bar{M}^{n+2}, k \leq n$, where $\bar{M}^{n+2}$ is a Brinkmann spacetime with $\xi^{b}=d U$, factorizes through a characteristic hypersurface if and only if the shape operator associated to $\xi^{\perp}$ satisfies $A_{\xi^{\perp}}=0$ and the function $U \circ \psi$ has a critical point.

In the compact case, we have, Corollary 2.6 ,

A spacelike submanifold $\psi: \Sigma^{k} \rightarrow \bar{M}^{n+2}, k \leq n$, where $\bar{M}^{n+2}$, is a Brinkmann spacetime with $\xi^{b}=d U$, factorizes through a characteristic hypersurface if $\mathbf{H}=f \xi^{\perp}$, for some $f \in C^{\infty}\left(\Sigma^{k}\right)$.

Section 3 mainly concerns with the search of sufficient conditions to ensure that a spacelike submanifold factorizes through a characteristic hypersurface of a Brinkmann spacetime. By means of Proposition 3.1, Theorem 3.3 deals with the compact case as follows,

Let $\psi: \Sigma^{k} \rightarrow \bar{M}^{n+2}$ be a compact spacelike submanifold in a Brinkmann spacetime $\bar{M}^{n+2}, k \leq n$, with trace $\left(A_{\xi^{\perp}}\right)=0$ (in particular with $\mathbf{H}=0$ ). If the Ricci tensor Ric of $\Sigma^{k}$ satisfies $\operatorname{Ric}\left(\xi^{\top}, \xi^{\top}\right) \geq 0$, then $\xi^{\top}$ is parallel. In addition, if Ric is positive definite at some point $p \in \Sigma^{k}$ or $\Sigma^{k}$ is simply connected, then $\xi^{\top}=0$ and $\Sigma^{k}$ factorizes through a characteristic hypersurface of $\bar{M}^{n+2}$.

In the non-compact complete case, as an application of classical results by Yau [27], we obtain, Theorem 3.6,

Let $\psi: \Sigma^{k} \rightarrow \bar{M}^{n+2}$ be a complete spacelike submanifold in a Brinkmann spacetime $\bar{M}^{n+2}, k \leq n$. If $\left\|\xi^{\top}\right\|^{2} \in L^{p}\left(\Sigma^{k}\right)$ for some $1<p<\infty, \mathbf{H}=0$ and Ric $\geq 0$, then $\xi^{\top}$ is a parallel vector field on $\Sigma^{k}$. In particular, if $\operatorname{Vol}\left(\Sigma^{k}\right)=\infty$, then we get $\xi^{\top}=0$ and, therefore, $\Sigma^{k}$ factorizes through a characteristic hypersurface of $\bar{M}^{n+2}$.

The remainder of Section 3 is devoted to give several results for spacelike surfaces $\Sigma^{2}$ in a Brinkmann spacetime $\bar{M}^{n+2}$. Lemma 3.8 provides a formula which relates the Gauss curvature $K$ of $\Sigma^{2}$ with the smooth function $\left\|\xi^{\top}\right\|^{2}$, when $\operatorname{trace}\left(A_{\xi}^{\perp}\right)=0$ holds on $\Sigma^{2}$. By means of this formula, Corollary 3.9 shows that a spacelike surface in a Brinkmann spacetime with $\tau>0$ and $\operatorname{trace}\left(A_{\xi}^{\perp}\right)=0$ (in particular if $\mathbf{H}=0$ holds) is globally point-wise conformally flat. If in addition $\Sigma^{2}$ is compact and orientable, then it is a topological torus. Finally, Proposition 3.10 provides a condition to assert that a non-compact complete spacelike surface factorizes through a characteristic hypersurface of $\bar{M}^{n+2}$. 
In Section 4 we consider a relevant subfamily of Brinkmann spacetimes, the so called plane fronted wave spacetimes introduced in [8]. A plane fronted wave spacetime is a time oriented Lorentzian manifold $\left(\bar{M}^{n+2},\langle\rangle,\right)$ where $\bar{M}^{n+2}=\mathbb{R}^{2} \times M^{n}, M^{n}$ an $n$-dimensional manifold, and Lorentzian metric

$$
\langle,\rangle=H(u, x) d u \otimes d u+d u \otimes d v+d v \otimes d u+g_{M},
$$

where $(u, v)$ are the natural coordinates on $\mathbb{R}^{2}$ and $H(u, x)$ is a (independent of $v$ ) smooth function on $\mathbb{R}^{2} \times M^{n}$ and $g_{M}$ a Riemannian metric on $M^{n}$. Since a plane fronted wave spacetime is topologically a product manifold, it possesses two distinguished smooth functions, namely, $u, v: \mathbb{R}^{2} \times M^{n} \rightarrow \mathbb{R}$, the canonical projections onto the first and second slots of $\mathbb{R}^{2}$. The parallel lightlike vector field is now $\partial_{v}:=\partial / \partial v$ and characteristic hypersurfaces are given by $u=u_{0}$, i.e., the level hypersurfaces of the function $u$. In this case, we have $\partial_{v}^{b}=d u$. Thus, for a codimension two spacelike submanifold $\Sigma^{n}$ in a plane fronted wave spacetime $\bar{M}^{n+2}$, Lemma 2.4 specializes to $\Delta(u \circ \psi)=k\left\langle\mathbf{H}, \partial_{v}\right\rangle$ and a formula for $\Delta(v \circ \psi)$ is given in (4.6).

Given any spacelike submanifold $\psi: \Sigma^{n} \rightarrow \bar{M}^{n+2}$ through a characteristic hypersurface $u=u_{0}$ in a plane fronted wave spacetime $\bar{M}^{n+2}$, the map $\pi_{M} \circ \psi: \Sigma^{n} \rightarrow M^{n}$, where $\pi_{M}: \bar{M}^{n+2} \rightarrow M^{n}$ is the natural projection, is a local isometry, Lemma 4.2. Thus, the local intrinsic geometry of $\Sigma^{n}$ is completely determined by the one of $M^{n}$. Even more, if $\Sigma^{n}$ is assumed to be complete, then $\pi_{M} \circ \psi: \Sigma^{n} \rightarrow M^{n}$ is a Riemannian covering map. In particular, if $M^{n}$ is simply connected, then $\pi_{M} \circ \psi$ is a global isometry, Proposition 4.6. These properties motivate that we restrict our attention to the extrinsic geometry of such spacelike submanifolds. In fact, in that direction we show that the second fundamental form $\amalg$ of a spacelike submanifold $\psi: \Sigma^{n} \rightarrow \bar{M}^{n+2}$ through a characteristic hypersurface satisfies $\operatorname{Im}(\amalg) \subset \operatorname{Span}\left(\partial_{v}\right)$. This fact permits to get an explicit formula for the corresponding mean curvature vector field $\mathbf{H}$ in 4.12 as follows,

$$
\mathbf{H}=\frac{1}{n} \Delta(v \circ \psi) \partial_{v} .
$$

As a direct consequence, Theorem 4.8 states

\section{There exists no compact codimension two spacelike submanifold in a plane fronted wave} spacetime with everywhere causal mean curvature vector field.

Note that, from equation (4.12), one deduces that the converse of Corollary 2.6 holds for compact spacelike submanifolds in a plane fronted wave spacetime. Moreover, formula (4.12) shows that the mean curvature vector field may be identically zero for codimension two spacelike submanifolds through a characteristic hypersurface in a plane fronted wave spacetime. This fact contrasts with the situation for spacelike submanifolds through a light cone in the Lorentz-Minkowski spacetime [3], [15] and in the de Sitter spacetime [2]. Indeed, the mean curvature vector field never vanishes for spacelike submanifolds in these cases. 
Next, we introduce in Section 4 the notion of spacelike graph through a characteristic hypersurface $u=u_{0}$ in a plane fronted wave spacetime as follows,

$$
\Sigma_{u_{0}}^{n}(h)=\left\{\left(u_{0}, h(x), x\right) \in \mathbb{R} \times \mathbb{R} \times M^{n}: x \in \Omega\right\},
$$

for a function $h \in C^{\infty}(\Omega)$, being $\Omega$ is an open domain of $M^{n}$, The graph $\Sigma_{u_{0}}^{n}(h)$ is a codimension two embedded spacelike submanifold for any $h$ and $u_{0}$. When $h$ is a constant $v_{0}$, the corresponding entire spacelike graph is the front of wave given by $u=u_{0}$ and $v=v_{0}$. Each front of wave is a totally geodesic spacelike submanifold in the plane fronted wave spacetime, and it may be interpreted as an idealized gravitational wave [6].

The spacelike graph $\Sigma_{u_{0}}^{n}(h)$ is indeed a spacelike hypersurface of the characteristic hypersurface $u=u_{0}$ of a plane fronted wave spacetime $\bar{M}^{n+2}$. The metric of $u=u_{0}$ is degenerate of rank $n$. The geometric behaviour of this kind of graphs differs to the well-known one of spacelike hypersurfaces in a spacetime First of all, no assumption is needed on $h \in C^{\infty}\left(M^{n}\right)$ to ensure that the corresponding graph $\Sigma_{u_{0}}^{n}(h)$ is spacelike. Secondly, the volume of a compact spacelike graph does not depend on the function used in its definition. Indeed, the induced metric on $M^{n}$ via the natural embedding (4.14) equals to the original metric $g_{M}$ independently of $h \in C^{\infty}\left(M^{n}\right)$. Therefore, the volume variational problem for this kind of spacelike graphs has no sense (compare with [5], [10], [19] and [20] for instance).

Taking into account that the mean curvature vector field of a spacelike graph $\Sigma_{u_{0}}^{n}(h)$ is 4.16 ,

$$
\mathbf{H}=\frac{1}{n}(\Delta h) \partial_{v}
$$

we get immediately,

If $M^{n}$ is compact, then the only entire spacelike graphs $\Sigma_{u_{0}}^{n}(h)$ which satisfy $\mathbf{H}=0$ are the front of waves $h=v_{0}, u=u_{0}$.

The paper ends with two uniqueness results for codimension two spacelike submanifolds in a plane fronted wave spacetime. In the compact case, Theorem 4.10 states,

A compact codimension two spacelike submanifold $\psi: \Sigma^{n} \rightarrow \bar{M}^{n+2}$, in a plane fronted wave spacetime $\bar{M}^{n+2}$, with signed $\left\langle\mathbf{H}, \partial_{v}\right\rangle$, factorizes through a characteristic hypersurface $u=u_{0}$ and $\Sigma^{n}$ is a Riemannian covering space of $M^{n}$ (in particular, $M^{n}$ is compact). If, in addition $\mathbf{H}=0$, then $\psi\left(\Sigma^{n}\right)$ is a (totally geodesic) front of wave.

Finally, Theorem 4.13) deals with the complete case. Now we impose a new assumption on the plane fronted wave spacetime $\bar{M}^{n+2}$, namely, the Timelike Convergence Condition, $\overline{\operatorname{Ric}}(X, X) \geq 0$ for every timelike tangent vector $X$, where $\overline{\operatorname{Ric}}$ is the Ricci tensor of $\bar{M}^{n+2}$. This energy condition may be seen as a mathematical translation that gravity on average attracts, and it also holds when the Einstein field equation (with zero cosmological constant) is satisfied for a realistic stress-energy tensor [21]. 
Consider a complete codimension two spacelike submanifold $\psi: \Sigma^{n} \rightarrow \bar{M}^{n+2}$, in a plane fronted wave spacetime $\bar{M}^{n+2}$, with $\mathbf{H}=0$ and that factorizes through a characteristic hypersurface $u=u_{0}$. If $\bar{M}^{n+2}$ satisfies the Timelike Convergence Condition and the function $v \circ \psi$ is bounded from above or from below, then $\psi\left(\Sigma^{n}\right)$ is a (totally geodesic) front of wave.

\section{Preliminaries and first results}

Consider a (connected) Lorentzian manifold $\left(\bar{M}^{n+2},\langle\rangle,\right)$ featuring a (globally defined) lightlike vector field $\xi$ that is parallel with respect to its Levi-Civita connection. Such a Lorentzian manifold must be time-orientable. Indeed, for any $p \in \bar{M}^{n+2}$ the future cone at $p$, [14, p.143], can defined as the one such that $\xi_{p}$ lies in its closure in $T_{p} \bar{M}$. In this way, we have a time orientation in $\left(\bar{M}^{n+2},\langle\rangle,\right)$ (see [14, p.145], for instance). A Lorentzian manifold which admits a lightlike and parallel vector field is called a Brinkmann spacetime.

A Brinkmann spacetime $\bar{M}^{n+2}$ has a natural distribution $D_{\xi}^{\perp}=\{X \in \mathfrak{X}(\bar{M}):\langle X, \xi\rangle=0\}$. For $X, Y \in D_{\xi}^{\perp}$, we have $\left\langle\bar{\nabla}_{X} Y, \xi\right\rangle=0$, where $\bar{\nabla}$ is the Levi-Civita connection of $\langle$,$\rangle , in particular$ $[X, Y] \in D_{\xi}^{\perp}$. Hence, the classical Frobenius theorem says that $D_{\xi}^{\perp}$ is integrable. Even more, each leaf $L_{\alpha}$ of $D_{\xi}^{\perp}$ is autoparallel. Moreover, taking into account that $\bar{\nabla}$ is symmetric, each $L_{\alpha}$ is totally geodesic with respect to $\bar{\nabla}$, i.e, if $\gamma$ is a geodesic of $\bar{M}^{n+2}$ starting tangent to $L_{\alpha}$, then $\gamma$ remains every time in $L_{\alpha}$. On the other hand, the fact $\bar{\nabla} \xi=0$ gives $\bar{R}(X, Y) \xi=0$ and $\overline{\operatorname{Ric}}(X, \xi)=0$, for all $X, Y \in \mathfrak{X}(\bar{M})$, where $\bar{R}$ and $\overline{\text { Ric }}$ are the curvature tensor and the Ricci tensor of $\bar{M}^{n+2}$, respectively.

It can be shown (see for instance [24]) that the metric $\langle$,$\rangle has the following description in local$ coordinates $\left\{\mathcal{O},\left(u, v, \mathbf{x}=\left(x_{1}, \cdots, x_{n}\right)\right)\right\}$,

$$
H(u, \mathbf{x}) d u \otimes d u+d u \otimes d v+d v \otimes d u+\sum_{i, j=1}^{n} g_{i j}(u, \mathbf{x}) d x_{i} \otimes d x_{j},
$$

where $H$ is a smooth function with no required sign. In this local picture, the parallel lightlike vector field $\xi$ coincides with the coordinate vector field $\partial_{v}$ on the open subset $\mathcal{O}$ of $\bar{M}^{n+2}$. The leaves of $D_{\partial_{v}}^{\perp}$ are the characteristic hypersurfaces $u=u_{0}$ and the map $\left(u_{0}, v, \mathbf{x}\right) \mapsto\left(u_{1}, v, \mathbf{x}\right)$ states that, at least locally, two leaves of $D_{\partial}^{\perp}$ are isometric.

Let $\psi: \Sigma^{k} \rightarrow \bar{M}^{n+2}$ be a spacelike submanifold in a Brinkmann spacetime $\bar{M}^{n+2}$, i.e., $\Sigma^{k}$ is a (connected) $k(\geq 2)$-dimensional smooth manifold and $\psi$ is a (globally defined) smooth immersion whose induced metric $\psi^{*}\langle$,$\rangle , as well denoted by \langle$,$\rangle , is Riemannian; in other words, \psi$ is a spacelike immersion. Thus, for each $p \in \Sigma^{k}$ there is an open neighbourhood $\Omega$ of $p$ such that $\psi_{\left.\right|_{\Omega}}: \Omega \rightarrow \bar{M}^{n+2}$ is a spacelike embedding. Consequently, $\Omega$ and $\psi(\Omega) \subset \bar{M}^{n+2}$ are naturally identifiable, and, as expected, $\psi$ is locally a spacelike embedding.

For a given vector field $V$ along the immersion $\psi$, we denote by $V^{\top} \in \mathfrak{X}\left(\Sigma^{k}\right)$ and $V^{\perp} \in \mathfrak{X}^{\perp}\left(\Sigma^{k}\right)$ the tangent and normal vector fields on $\Sigma^{k}$, respectively, naturally obtained from $V$. In particular, for 
$V=\xi \circ \psi \equiv \xi$, where $\xi$ is the parallel lightlike vector field on $\bar{M}^{n+2}$, we have,

$$
0=\langle\xi, \xi\rangle=\left\langle\xi^{\top}, \xi^{\top}\right\rangle+\left\langle\xi^{\perp}, \xi^{\perp}\right\rangle .
$$

Hence, taking into account that $\Sigma^{k}$ is spacelike, $\xi^{\perp}$ does not vanish at any point of $\Sigma^{k}$. Even more, formula 2.2 gives that the normal vector field $\xi^{\perp}$ is causal (i.e., timelike or lightlike) at any point of $\Sigma^{k}$. Moreover, the vector field $\xi^{\top}$ vanishes identically if and only if $\psi\left(\Sigma^{k}\right)$ is contained in a characteristic hypersurface $L_{\alpha}$ of $\bar{M}^{n+2}$. If a spacelike submanifold $\psi: \Sigma^{k} \rightarrow \bar{M}^{n+2}$ satisfies $\psi\left(\Sigma^{k}\right) \subset L_{\alpha}$, for some $L_{\alpha}$, we can write $\psi=i \circ \tilde{\psi}$ where $\tilde{\psi}: \Sigma^{k} \rightarrow L_{\alpha}, \tilde{\psi}(p):=\psi(p)$, for all $p \in \Sigma^{k}$ and $i: L_{\alpha} \rightarrow \bar{M}^{n+2}$ is the inclusion map. Thus, we will say that $\Sigma^{k}$ factorizes through a characteristic hypersurface of $\bar{M}^{n+2}$ in this case.

Remark 2.1. When $\Sigma^{n+1}$ is a spacelike hypersurface in $\bar{M}^{n+2}$, the decomposition (2.2) gives that $\xi^{\top}$ is a nowhere vanishing vector field on $\Sigma^{k}$. Hence, if $\Sigma^{n+1}$ is assumed to be compact, then its Euler number vanishes.

Let us take $V, W \in \mathfrak{X}\left(\Sigma^{k}\right)$ and $\zeta \in \mathfrak{X}^{\perp}\left(\Sigma^{k}\right)$. Then, the Gauss and Weingarten formulae are respectively written as

$$
\bar{\nabla}_{V} W=\nabla_{V} W+\amalg(V, W),
$$

and

$$
\bar{\nabla}_{V} \zeta=-A_{\zeta} V+\nabla_{V}^{\perp} \zeta
$$

Here, $\nabla$ denotes the Levi-Civita connection of $\Sigma^{k}$, $\amalg$ the second fundamental form of the spacelike submanifold, $A_{\zeta}$ the shape operator associated to the normal vector field $\zeta$ and $\nabla^{\perp}$ the normal connection. The shape operator $A_{\zeta}$ is related to the second fundamental form $\amalg$ by

$$
\left\langle A_{\zeta}(V), W\right\rangle=\langle\amalg(V, W), \zeta\rangle,
$$

for all $V, W \in \mathfrak{X}\left(\Sigma^{k}\right)$. As usual, we define the mean curvature vector field by

$$
\mathbf{H}=\frac{1}{k} \operatorname{trace}_{\langle,\rangle} \amalg,
$$

where trace $\left\langle_{,\rangle}\right.$is the trace of $\amalg$ with respect to the metric induced via $\psi$. From $(2.5)$, we have,

$$
\operatorname{trace}\left(A_{\zeta}\right)=k\langle\mathbf{H}, \zeta\rangle .
$$

Therefore, if $\mathbf{H}$ is lightlike everywhere then $\operatorname{trace}\left(A_{\mathbf{H}}\right)=0$.

From formulae (2.3) and (2.4) we obtain

$$
0=\bar{\nabla}_{V} \xi=\bar{\nabla}_{V} \xi^{\top}+\bar{\nabla}_{V} \xi^{\perp}=\nabla_{V} \xi^{\top}+\amalg\left(V, \xi^{\top}\right)-A_{\xi^{\perp}} V+\nabla_{V}^{\perp} \xi^{\perp},
$$

for every $V \in \mathfrak{X}\left(\Sigma^{k}\right)$. Thus, we have

$$
A_{\xi^{\perp}} V=\nabla_{V} \xi^{\top},
$$


and

$$
\amalg\left(V, \xi^{\top}\right)=-\nabla_{V}^{\perp} \xi^{\perp} .
$$

Directly from (2.6) and (2.7), we get

$$
\operatorname{div}\left(\xi^{\top}\right)=k\langle\mathbf{H}, \xi\rangle
$$

If $\Sigma^{k}$ is assumed to be compact, formula 2.9 gives

$$
\int_{\Sigma^{k}}\langle\mathbf{H}, \xi\rangle d \mu_{\langle,\rangle}=0
$$

Of course, the causal character of $\mathbf{H}_{p}$ may change with $p \in \Sigma^{k}$. In the special, but important, case of a codimension two spacelike submanifold $\Sigma^{n}$, we will say that it is trapped if $\mathbf{H}$ is timelike [17], marginally trapped if $\mathbf{H}$ is lightlike, and $\Sigma^{n}$ is weakly trapped if $\mathbf{H}$ is causal.

Remark 2.2. Consider a weakly trapped submanifold $\psi: \Sigma^{n} \rightarrow \bar{M}^{n+2}$ of a Brinkmann spacetime $\bar{M}^{n+2}$ and assume $\Sigma^{n}$ is compact. Taking into account that $\xi$ is lightlike and consequently, the function $\langle\mathbf{H}, \xi\rangle$ satisfies either $\langle\mathbf{H}, \xi\rangle \leq 0$ or $\langle\mathbf{H}, \xi\rangle \geq 0$, everywhere, we get $\langle\mathbf{H}, \xi\rangle=0$ directly from 2.10 . Therefore, $\mathbf{H}$ must be collinear to $\xi$ at every point of $\Sigma^{n}$ [14, p.155] ( $\Sigma^{n}$ becomes in fact marginally trapped). In particular, we have $\xi=\xi^{\perp}$ on $\Sigma^{n}$. Thus, we have obtained (compare with [4, Remark 4.2], [13]),

Any compact weakly trapped submanifold $\psi: \Sigma^{n} \rightarrow \bar{M}^{n+2}$ of a Brinkmann spacetime becomes marginally trapped and factorizes through a characteristic hypersurface of $\bar{M}^{n+2}$.

On the other hand, if it is assumed either $\langle\mathbf{H}, \xi\rangle \geq 0$ or $\leq 0$ everywhere (instead $\mathbf{H}$ is causal), we also arrive to $\langle\mathbf{H}, \xi\rangle=0$ thanks to 2.10 . Using again that $\xi$ is lightlike, we conclude that $\mathbf{H}$ is at any point spacelike or zero. Thus, $\langle\mathbf{H}, \mathbf{H}\rangle \geq 0$ everywhere on $\Sigma^{n}$.

Next, we give several formulas involving the Ricci tensor of a spacelike submanifold $\Sigma^{k}$ (of any dimension) in $\bar{M}^{n+2}$. Making use of (2.7), we obtain for the curvature tensor $R$ of $\Sigma^{k}$,

$$
R(V, W) \xi^{\top}=\left(\nabla_{V} A_{\xi^{\perp}}\right) W-\left(\nabla_{W} A_{\xi^{\perp}}\right) V
$$

for all $V, W \in \mathfrak{X}\left(\Sigma^{k}\right)$. Now, we put $W=\xi^{\top}$ in previous formula and, after taking trace in $V$, we get

$$
\operatorname{div}\left(A_{\xi^{\perp}} \xi^{\top}\right)=\operatorname{Ric}\left(\xi^{\top}, \xi^{\top}\right)+\operatorname{trace}\left(A_{\xi^{\perp}}^{2}\right)+\xi^{\top}\left(\operatorname{trace}\left(A_{\xi^{\perp}}\right)\right) .
$$

Taking into account (2.7), 2.6 and 2.9), formula (2.12) is rewritten as

$$
\operatorname{div}\left(A_{\xi^{\perp}} \xi^{\top}\right)=\operatorname{Ric}\left(\xi^{\top}, \xi^{\top}\right)+\operatorname{trace}\left(\nabla^{2} \xi^{\top}\right)+\xi^{\top}\left(\operatorname{div}\left(\xi^{\top}\right)\right),
$$

where trace $\left(\nabla^{2} \xi^{\top}\right)$ means trace $\left(V \mapsto \nabla_{\nabla_{V} \xi^{\top}} \xi^{\top}\right)$. 
Remark 2.3. Note that 2.13) is none other that the classical Bochner formula, which holds true for any vector field on a Riemannian manifold (see for instance [25]).

$$
\operatorname{div}\left(\nabla_{V} V\right)=\operatorname{Ric}(V, V)+\operatorname{trace}\left(\nabla^{2} V\right)+V(\operatorname{div}(V))
$$

for $V=\xi^{\top} \in \mathfrak{X}\left(\Sigma^{k}\right)$.

Now, we let $\tau$ be the smooth function $\left\|\xi^{\top}\right\|^{2}$ on $\Sigma^{k}$. Using 2.7), we get the following expression for its gradient

$$
\nabla \tau=2 A_{\xi^{\perp}} \xi^{\top}
$$

Therefore, formula 2.12 gives

$$
\frac{1}{2} \Delta \tau=\operatorname{Ric}\left(\xi^{\top}, \xi^{\top}\right)+\operatorname{trace}\left(A_{\xi^{\perp}}^{2}\right)+\xi^{\top}\left(\operatorname{trace}\left(A_{\xi^{\perp}}\right)\right)
$$

with the advantage that now the left hand side is not only a divergence but also the Laplacian of a smooth function on $\Sigma^{k}$.

Now note that, by Poincaré's lemma, we always have $\xi^{b}=d U$, where $\xi^{b}=\langle\xi,-\rangle$, for a smooth function $U$ at least locally on $\bar{M}^{n+2}$. Of course, if $\bar{M}^{n+2}$ is simply connected then equality $\xi^{b}=d U$ holds on all $\bar{M}^{n+2}$. Moreover, in this case the smooth function $U$ is determined by $\xi$ up to an additive constant from the connectedness of $\bar{M}^{n+2}$. Thus, the level sets of $U$ are the leaves of the foliation $D_{\xi}^{\perp}$. Hence, a spacelike submanifold $\psi: \Sigma^{k} \rightarrow \bar{M}^{n+2}, k \leq n$, factorizes through a characteristic hypersurface of $\bar{M}^{n+2}$ if and only if $U \circ \psi$ is constant.

Lemma 2.4. If $\psi: \Sigma^{k} \rightarrow \bar{M}^{n+2}$ is a spacelike submanifold into a Brinkmann spacetime $\bar{M}^{n+2}$ with $\xi^{b}=d U$, then

$$
\Delta(U \circ \psi)=k\langle\mathbf{H}, \xi\rangle
$$

Proof. Taking into account that $\bar{\nabla} U=\xi$ on $\bar{M}^{n+2}$, we get for the gradient of $U \circ \psi$ on $\Sigma^{k}$,

$$
\nabla(U \circ \psi)=\xi^{\top}
$$

From 2.9), previous formula gives the announced result.

Formula (2.7) implies $A_{\xi^{\perp}}=A_{\xi}=0$ for a spacelike submanifold through a characteristic hypersurface of $\bar{M}^{n+2}$. The following result gives a partial converse of this fact.

Corollary 2.5. A spacelike submanifold $\psi: \Sigma^{k} \rightarrow \bar{M}^{n+2}, k \leq n$, where $\bar{M}^{n+2}$ is a Brinkmann spacetime with $\xi^{b}=d U$, factorizes through a characteristic hypersurface if and only if the shape operator associated to $\xi^{\perp}$ satisfies $A_{\xi^{\perp}}=0$ and the function $U \circ \psi$ has a critical point.

Proof. Formula 2.17) and the assumption $A_{\xi^{\perp}}=0$ imply that $\nabla(U \circ \psi)$ is a parallel vector field on $\Sigma^{k}$ thanks to 2.7$)$. Thus, from the existence of a critical point for $U \circ \psi$, we get that $\nabla(U \circ \psi)$ vanishes identically. The converse is clear. 
Formula 2.6 gives $\langle\mathbf{H}, \xi\rangle=0$ for a spacelike submanifold through a characteristic hypersurface of $\bar{M}^{n+2}$. The converse is true in the case $\xi^{b}=d U$, in particular, we derive the following direct consequence of 2.16,

Corollary 2.6. A spacelike submanifold $\psi: \Sigma^{k} \rightarrow \bar{M}^{n+2}, k \leq n$, where $\bar{M}^{n+2}$, is a Brinkmann spacetime with $\xi^{b}=d U$, factorizes through a characteristic hypersurface if $\mathbf{H}=f \xi^{\perp}$, for some $f \in C^{\infty}\left(\Sigma^{k}\right)$.

Finally, observe that if $\xi^{b}=d U$ globally, we have $\tau=\|\nabla(U \circ \psi)\|^{2}, \operatorname{trace}\left(A_{\xi^{\perp}}^{2}\right)=\|\operatorname{Hess}(U \circ \psi)\|^{2}$, the squared length of the Hessian of $U \circ \psi$ on $\Sigma$ and $\xi^{\top}\left(\operatorname{trace}\left(A_{\xi^{\perp}}\right)\right)=\langle\nabla(U \circ \psi), \nabla \Delta(U \circ \psi)\rangle$, and thus, (2.15) becomes in this case

$$
\begin{aligned}
\frac{1}{2} \Delta\|\nabla(U \circ \psi)\|^{2} & =\operatorname{Ric}(\nabla(U \circ \psi), \nabla(U \circ \psi))+ \\
& +\|\operatorname{Hess}(U \circ \psi)\|^{2}+\langle\nabla(U \circ \psi), \nabla \Delta(U \circ \psi)\rangle,
\end{aligned}
$$

namely, the Bochner-Lichnerowicz formula for the function $U \circ \psi$ on $\Sigma^{k}$, [25].

\section{Sufficient conditions for a spacelike submanifold factorizes through a characteristic hypersurface}

If a spacelike submanifold $\psi: \Sigma^{k} \rightarrow \bar{M}^{n+2}$ in a Brinkmann spacetime $\bar{M}^{n+2}$ factorizes through a characteristic hypersurface of $\bar{M}^{n+2}$, then $k \leq n$. In what follows, we assume $k \leq n$ unless otherwise is stated. Specially, we will pay special attention to the case $k=n$, i.e., to codimension two spacelike submanifolds.

Proposition 3.1. For any compact spacelike submanifold $\psi: \Sigma^{k} \rightarrow \bar{M}^{n+2}$ in a Brinkmann spacetime $\bar{M}^{n+2}$ we have

$$
\int_{\Sigma^{k}}\left[\operatorname{Ric}\left(\xi^{\top}, \xi^{\top}\right)+\xi^{\top}\left(\operatorname{trace}\left(A_{\xi^{\perp}}\right)\right)\right] d \mu_{\langle,\rangle} \leq 0,
$$

with equality if and only if the vector field $\xi^{\top}$ is parallel. Moreover, if $\bar{M}^{n+2}$ is simply connected then equality in (3.1) holds if and only if $\Sigma^{k}$ factorizes through a characteristic hypersurface of $\bar{M}^{n+2}$.

Proof. The inequality is a direct consequence of (2.12), making use of the divergence theorem and the fact trace $\left(A_{\xi^{\perp}}^{2}\right) \geq 0$. Moreover, the equality holds if and only if $A_{\xi^{\perp}}=0$, i.e., if and only if $\xi^{\top}$ is parallel, making use of 2.7).

If $\Sigma^{k}$ factorizes through a characteristic hypersurface of $\bar{M}^{n+2}$, then $\xi^{\top}=0$, that trivially gives the equality in 3.1). Conversely, if equality holds in 3.1 ) then the vector field $\xi^{\top}=\nabla(U \circ \psi)$ is parallel, where $\xi^{b}=d U$. In this case $\xi^{\top}$ vanishes at a critical point of $U \circ \psi$ on $\Sigma^{k}$ compact. Therefore, $U \circ \psi$ is constant, which means that $\Sigma^{k}$ factorizes through a characteristic hypersurface of $\bar{M}^{n+2}$. 
Remark 3.2. In particular when $\Sigma^{k}$ is orientable with non-zero Euler number, the equality in Proposition 3.1 implies that $\Sigma^{k}$ factorizes through a characteristic hypersurface of $\bar{M}^{n+2}$.

Now, we will deal with the assumption

$$
\operatorname{trace}\left(A_{\xi^{\perp}}\right)=0
$$

which has a clear variational interpretation. Namely, consider the $k$-volume functional acting on compactly supported spacelike variations of $\psi$ along the normal direction defined by $\xi^{\perp}$. The spacelike immersion $\psi$ is a critical point of this functional if and only if the component of the mean curvature vector field in the direction of $\xi^{\perp}$ identically vanishes, equivalently if $\left\langle\mathbf{H}, \xi^{\perp}\right\rangle=0$. But, making use of (2.6), this condition is equivalent to $(3.2)$. Thus, normal vector field $\xi^{\perp}$ is said to be stationary in this case. Clearly, condition (3.2) is weaker than $\mathbf{H}=0$. From (2.7), every spacelike submanifold through a characteristic hypersurface of $\bar{M}^{n+2}$ satisfies $A_{\xi^{\perp}}=0$ and, therefore, (3.2) in particular.

Theorem 3.3. Let $\psi: \Sigma^{k} \rightarrow \bar{M}^{n+2}$ be a compact spacelike submanifold in a Brinkmann spacetime $\bar{M}^{n+2}$ with stationary normal vector field $\xi^{\perp}$ (in particular with $\left.\mathbf{H}=0\right)$. If $\operatorname{Ric}\left(\xi^{\top}, \xi^{\top}\right) \geq 0$, then $\xi^{\top}$ is parallel. In addition, if Ric is positive definite at some point $p \in \Sigma^{k}$ or $\Sigma^{k}$ is simply connected, we have $\xi^{\top}=0$ and $\Sigma^{k}$ factorizes through a characteristic hypersurface of $\bar{M}^{n+2}$.

Proof. First of all, the second integrand term in 3.1 vanishes from the assumption trace $\left(A_{\xi^{\perp}}\right)=0$ and then 3.1 becomes

$$
\int_{\Sigma^{k}} \operatorname{Ric}\left(\xi^{\top}, \xi^{\top}\right) d \mu_{\langle,\rangle} \leq 0 .
$$

Hence, previous inequality is indeed an equality, that implies $\operatorname{Ric}\left(\xi^{\top}, \xi^{\top}\right)=0$ on $\Sigma^{k}$. Moreover, we have $A_{\xi^{\perp}}=0$ and thus, formula $\sqrt{2.7)}$ implies that $\xi^{\top}$ is a parallel.

If Ric is positive definite at a point $p \in \Sigma^{k}$, the equality $\operatorname{Ric}\left(\xi^{\top}(p), \xi^{\top}(p)\right)=0$ gives rise to $\xi^{\top}(p)=0$. Being $\xi^{\top}$ parallel, we arrive to $\xi^{\top}=0$ everywhere on $\Sigma^{k}$. On the other hand, if $\Sigma^{k}$ is simply connected, we also get $\xi^{\top}=0$ since the vector field has necessarily a zero. In both cases, $\Sigma^{k}$ factorizes through a characteristic hypersurface of $\bar{M}^{n+2}$.

Remark 3.4. Under the assumptions of Theorem 3.3, the constancy of $\tau$ does not imply $\tau=0$, in general. In fact, let $\psi: \Sigma^{k} \rightarrow \bar{M}^{n+2}$ be a spacelike submanifold into a Brinkmann spacetime such that $\xi^{b}=d U$. Assume trace $\left(A_{\xi^{\perp}}\right)=0$ and the Ricci tensor of $\Sigma^{k}$ is positive semi-definite. In this case, the constancy of $\tau:=\|\nabla(U \circ \psi)\|^{2}$ implies, making use of $(2.18)$ that $\operatorname{Hess}(U \circ \psi)=0$. When $\Sigma^{k}=\mathbb{R}^{k}$, this means $(U \circ \psi)\left(x_{1}, \cdots, x_{k}\right)=\sum_{i} a_{i} x_{i}+b$, for some $a_{i}, b \in \mathbb{R}$.

Remark 3.5. In order to obtain a local result, the compactness assumption in Theorem 3.3 may be modified to obtain the following result,

Let $\psi: \Sigma^{k} \rightarrow \bar{M}^{n+2}$ be a spacelike submanifold in a Brinkmann spacetime $\bar{M}^{n+2}$ with stationary normal vector field $\xi^{\perp}$ (in particular with $\mathbf{H}=0$ ). Assume the Ricci tensor of 
$\Sigma^{k}$ is positive definite and there exists a relative maximum of $\tau$ at $p_{0} \in \Sigma^{k}$ (or $\tau$ attains a relative maximum at $p_{0} \in \Sigma^{k}$ and $\mathrm{Ric}_{p_{0}}$ is positive definite), then there exists an open neighbourhood of $p_{0}$ in $\Sigma^{k}$ that factorizes through a characteristic hypersurface of $\bar{M}^{n+2}$.

In fact, using $(2.15)$ we have

$$
0 \geq \frac{1}{2}(\Delta \tau)_{p_{0}}=\operatorname{Ric}\left(\xi_{p_{0}}^{\top}, \xi_{p_{0}}^{\top}\right)+\operatorname{trace}\left(A_{\xi^{\perp}}^{2}\right)_{p_{0}}
$$

therefore $\operatorname{Ric}\left(\xi_{p_{0}}^{\top}, \xi_{p_{0}}^{\top}\right) \leq-\operatorname{trace}\left(A_{\xi^{\perp}}^{2}\right)_{p_{0}} \leq 0$, however $\operatorname{Ric}\left(\xi_{p_{0}}^{\top}, \xi_{p_{0}}^{\top}\right) \geq 0$ by assumption. Hence $\operatorname{Ric}\left(\xi_{p_{0}}^{\top}, \xi_{p_{0}}^{\top}\right)=0$, which implies $\xi_{p_{0}}^{\top}=0$, that is $\tau\left(p_{0}\right)=0$ and therefore $\tau=0$ in an open neighbourhood of $p_{0}$.

In the non-compact and complete case, as an application of classical results by Yau in [27], we have,

Theorem 3.6. Let $\psi: \Sigma^{k} \rightarrow \bar{M}^{n+2}$ be a non-compact complete spacelike submanifold in a Brinkmann spacetime $\bar{M}^{n+2}$ with stationary normal vector field $\xi^{\perp}$ (in particular with $\mathbf{H}=0$ ) and positive semidefinite Ricci tensor. If $\tau \in L^{p}\left(\Sigma^{k}\right)$ for some $p$ such that $1<p<+\infty$ then, $\tau=0$ and $\Sigma^{k}$ factorizes through a characteristic hypersurface of $\bar{M}^{n+2}$.

Proof. From 2.15], we get $\Delta \tau \geq 0$. Being $\tau$ subharmonic, [27, Theorem 3] can be used to obtain $\tau$ is constant. Now, [27, Theorem 7] gives $\operatorname{Vol}\left(\Sigma^{k}\right)=+\infty$. Therefore, necessarily $\tau=0$, or equivalently $\xi^{\top}=0$.

Remark 3.7. In contrast with Theorem 3.3, we have not assumed here the existence of a point of $\Sigma^{k}$ where Ric is positive definite. If this assumption had been made then the corresponding argument in the proof of Theorem 3.3 could be performed to end the proof now.

We conclude this section giving several results for spacelike surfaces.

Lemma 3.8. Let $\psi: \Sigma^{2} \rightarrow \bar{M}^{n+2}$ be a spacelike surface in a Brinkmann spacetime $\bar{M}^{n+2}$ with stationary normal vector field $\xi^{\perp}$ (in particular with $\mathbf{H}=0$ ), then

$$
\tau \Delta \tau=2 K \tau^{2}+\|\nabla \tau\|^{2} .
$$

Proof. Under our assumptions, formula (2.15) reads as follows

$$
\Delta \tau=2 K \tau+2 \operatorname{trace}\left(A_{\xi^{\perp}}^{2}\right) .
$$

The hypothesis (3.2) and the Cayley-Hamilton theorem for $A_{\xi^{\perp}}$ imply that $A_{\xi^{\perp}}^{2}=-\operatorname{det}\left(A_{\xi^{\perp}}\right) I$, where $I$ stands for the identity transformation. Therefore, (3.4) gives

$$
\Delta \tau=2 K \tau-4 \operatorname{det}\left(A_{\xi^{\perp}}\right) .
$$

On the other hand, from formula (2.14) we obtain

$$
\|\nabla \tau\|^{2}=4\left\|A_{\xi^{\perp}} \xi^{\top}\right\|^{2}=4\left\langle A_{\xi^{\perp}}^{2} \xi^{\top}, \xi^{\top}\right\rangle=-4 \operatorname{det}\left(A_{\xi^{\perp}}\right) \tau .
$$

Hence, we get (3.3) from (3.5) and (3.6). 
Corollary 3.9. Let $\psi: \Sigma^{2} \rightarrow \bar{M}^{n+2}$ be a spacelike surface in a Brinkmann spacetime with $\tau>0$. If $\xi^{\perp}$ is stationary (in particular if $\mathbf{H}=0$ holds), then the induced metric on $\Sigma^{2}$ is globally point-wise conformally flat. If in addition $\Sigma^{2}$ is compact and orientable, then it is a topological torus.

Proof. From (3.3), it is followed

$$
\Delta \log \tau=\frac{\tau \Delta \tau-\|\nabla \tau\|^{2}}{\tau^{2}}=2 K
$$

which means that the metric $g^{*}:=\tau g$ is flat.

Hence, $\Sigma^{2}$ is a topological torus, whenever $\Sigma^{2}$ is compact and orientable. Alternatively, the (abstract) Gauss-Bonnet formula and 3.7) give that the Euler number of $\Sigma^{2}$ is zero.

Proposition 3.10. Let $\psi: \Sigma^{2} \rightarrow \bar{M}^{n+2}$ be a non-compact complete spacelike surface in a Brinkmann spacetime $\bar{M}^{n+2}$ with $K \geq 0$ and $\xi^{\perp}$ stationary (in particular if $\mathbf{H}=0$ holds). Assume $\tau$ is bounded from above. If there exists $p_{0} \in \Sigma^{2}$ such that $K\left(p_{0}\right)>0$, then $\Sigma^{2}$ factorizes through a characteristic hypersurface of $\bar{M}^{n+2}$.

Proof. From (3.5), the assumption $K \geq 0$ gives that $\tau$ is subharmonic. On the other hand, we know that a non-compact complete Riemannian surface with $K \geq 0$ is parabolic by a classical result of Ahlfors and Blanc-Fiala-Huber [12]. Thus, being $\tau$ subharmonic, bounded from above and $\Sigma^{2}$ parabolic, necessarily $\tau=\tau_{0}$ constant. Using again 3.5 we arrive to $\tau=0$, i.e., $\xi^{\top}=0$, concluding the proof.

\section{Codimension two spacelike submanifolds in a plane fronted wave spacetime}

A relevant subfamily of Brinkmann spacetimes consists of the so called plane fronted wave spacetimes, introduced in [8]. A plane fronted wave spacetime is a Lorentzian manifold $\left(\bar{M}^{n+2},\langle\rangle,\right)$ where $\bar{M}^{n+2}=\mathbb{R}^{2} \times M^{n}, M^{n}$ an $n$-dimensional manifold, and Lorentzian metric

$$
\langle,\rangle=H(u, x) d u \otimes d u+d u \otimes d v+d v \otimes d u+g_{M},
$$

where $(u, v)$ are the natural coordinates on $\mathbb{R}^{2}$ and $H(u, x)$ is a (independent of $v$ ) smooth function on $\mathbb{R}^{2} \times M^{n}$ and $g_{M}$ a Riemannian metric on $M^{n}$. The coordinate vector field $\partial_{v}$ is lightlike and parallel (see Lemma 4.1 below). Therefore, a plane fronted wave spacetime $\left(\bar{M}^{n+2},\langle\rangle,\right)$ is a Brinkmann spacetime.

A plane fronted wave spacetime is foliated by the characteristic hypersurfaces $u=u_{0}$. Indeed, if we take a coordinate neighbourhood $\left(V, x_{1}, \cdots, x_{n}\right)$ of $M^{n}$, the corresponding local expression of 4.1 on $\mathcal{O}=\mathbb{R}^{2} \times V$ specializes formula 2.1) with $g_{i j}\left(u, x_{1}, \cdots, x_{n}\right)=\left(g_{M}\right)_{i j}\left(x_{1}, \cdots, x_{n}\right)$. Observe that plane fronted wave spacetimes are natural generalizations of pp-wave spacetimes and gravitational plane wave spacetimes [6, Chap. 13], [8]. 
Let $\mathcal{L}\left(M^{n}\right)$ be the subspace of $\mathfrak{X}(\bar{M})$ consisting of the lifts to $\bar{M}^{n+2}$ of all vector fields on $M^{n}$. From now on, we denote by the same symbol a vector field in $\mathfrak{X}\left(M^{n}\right)$ and the corresponding lift in $\mathcal{L}\left(M^{n}\right)$. In a similar way, we simplify the notation by writing by the same symbol a function on $\mathbb{R}^{2}$ or $M^{n}$ and its corresponding lift to $\bar{M}^{n+2}$. The Levi-Civita connection $\bar{\nabla}$ of a plane fronted wave spacetime was given in [8] as follows,

Lemma 4.1. If $V, W \in \mathcal{L}\left(M^{n}\right) \subset \mathfrak{X}(\bar{M})$ and $\widetilde{\nabla}$ denotes the Levi-Civita connection of $\left(M^{n}, g_{M}\right)$, then we have,

i) $\bar{\nabla}_{\partial_{u}} \partial_{u}=\frac{1}{2}\left(\partial_{u} H \partial_{v}-\widetilde{\nabla} H_{u}\right)=\frac{1}{2}\left(\bar{\nabla} H-\widetilde{\nabla} H_{u}\right)$,

ii) $\bar{\nabla}_{V} \partial_{u}=\bar{\nabla}_{\partial_{u}} V=\frac{1}{2} g_{M}\left(\widetilde{\nabla} H_{u}, V\right) \partial_{v}$,

iii) $\bar{\nabla}_{V} W=\widetilde{\nabla}_{V} W$,

iv) $\bar{\nabla}_{\partial_{v}} \partial_{v}=\bar{\nabla}_{\partial_{v}} \partial_{u}=\bar{\nabla}_{\partial_{u}} \partial_{v}=\bar{\nabla}_{V} \partial_{v}=\bar{\nabla}_{\partial_{v}} V=0$,

where $\widetilde{\nabla} H_{u}$ denotes the gradient of $H_{u}$ on $M^{n}, H_{u}(x):=H(u, x)$, and $\bar{\nabla} H$ the gradient of $H$ on $\bar{M}^{n+2}$.

Every $w \in T_{(u, v, x)} \bar{M}^{n+2}$ admits the decomposition

$$
w=\left\langle w, \partial_{v}\right\rangle \partial_{u}+\left(\left\langle w, \partial_{u}\right\rangle-\left\langle w, \partial_{v}\right\rangle H\right) \partial_{v}+d \pi_{M}(w)
$$

where $\pi_{M}: \bar{M}^{n+2} \rightarrow M^{n}$ is the natural projection. To shorten notation, we have omitted the point $(u, v, x)$ in 4.2). From Lemma 4.1 and using the above decomposition, we get

$$
\bar{\nabla}_{w} \partial_{u}=\frac{1}{2}\left\langle w, \partial_{v}\right\rangle\left(\bar{\nabla} H-\widetilde{\nabla} H_{u}\right)+\frac{1}{2} g_{M}\left(\widetilde{\nabla} H_{u}, d \pi_{M}(w)\right) \partial_{v} .
$$

With the same notation as in Lemma 4.1, the non-necessarily vanishing components of the Riemann curvature tensor $\bar{R}$ of the metric 4.1 ) are,

$$
\bar{R}\left(V, \partial_{u}\right) \partial_{u}=-\frac{1}{2} \widetilde{\nabla}_{V} \widetilde{\nabla} H_{u} \quad \text { and } \quad \bar{R}\left(V, \partial_{u}\right) W=\frac{1}{2} \widetilde{\operatorname{Hess}}\left(H_{u}\right)(V, W) \partial_{v}
$$

where $\widetilde{\text { Hess }}$ stands for the Hessian tensor on $M^{n}$.

Denoting by $\overline{\mathrm{Ric}}$ and $\mathrm{Ric}_{M}$ the Ricci tensor of $\bar{M}^{n+2}$ and $M^{n}$, respectively, we have that the non-necessarily vanishing components of $\overline{\mathrm{Ric}}$ are

$$
\overline{\operatorname{Ric}}(V, W)=\operatorname{Ric}_{M}(V, W) \text { and } \overline{\operatorname{Ric}}\left(\partial_{u}, \partial_{u}\right)=-\frac{1}{2} \widetilde{\Delta} H_{u},
$$

where $\widetilde{\Delta}$ is the Laplacian operator on $M^{n}$. From these formulas it is easy to check that a plane fronted wave spacetime $\left(\bar{M}^{n+2},\langle\rangle,\right)$ satisfies the Timelike Convergence Condition (i. e., $\overline{\operatorname{Ric}}(T, T) \geq 0$ for every timelike vector $T$ ) if and only if,

$$
\widetilde{\Delta} H_{u} \leq 0 \text { and } \quad \operatorname{Ric}_{M} \geq 0 .
$$


Let $\psi: \Sigma^{n} \rightarrow \bar{M}^{n+2}$ be a codimension two spacelike submanifold in a plane fronted wave spacetime. We denote by $u, v: \bar{M}^{n+2}=\mathbb{R}^{2} \times M^{n} \rightarrow \mathbb{R}$ the standard projections onto the first and second slots of $\mathbb{R}^{2}$, respectively. Then, $d u=\partial_{v}^{b}$ and therefore, formula 2.16 specializes to

$$
\Delta(u \circ \psi)=n\left\langle\mathbf{H}, \partial_{v}\right\rangle .
$$

On the other hand, taking into account $\bar{\nabla} v=\partial_{u}-H \partial_{v}$, we obtain from 2.9

$$
\Delta(v \circ \psi)=\operatorname{div}\left(\partial_{u}^{\top}\right)-\partial_{v}^{\top}(H \circ \psi)-n(H \circ \psi)\left\langle\mathbf{H}, \partial_{v}\right\rangle .
$$

Next, the following result states that the intrinsic local geometry of a codimension two spacelike submanifold of a plane fronted wave spacetime through a characteristic hypersurface $u=u_{0}$ is completely determined by the one of $M^{n}$. Thus, to study such a spacelike submanifolds we must focus on their extrinsic geometry. Although this result was proven in [11], we give here a proof for the sake of completeness.

Lemma 4.2. Let $\psi: \Sigma^{n} \rightarrow \bar{M}^{n+2}$ be a codimension two spacelike submanifold in a plane fronted wave spacetime $\bar{M}^{n+2}$ which factorizes through a characteristic hypersurface $u=u_{0}$. Then, the map $\pi_{M} \circ \psi: \Sigma^{n} \rightarrow M^{n}$ is a local isometry.

Proof. Under our assumptions, we write the immersion $\psi=\left(u_{0}, h, \pi_{M} \circ \psi\right)$ with $h \in C^{\infty}(\Sigma)$. For all $w_{1}, w_{2} \in T_{p} M^{n}$ we have $d \psi_{p}\left(w_{i}\right)=\left(0, w_{i}(h), d\left(\pi_{M} \circ \psi\right)_{p}\left(w_{i}\right)\right), i=1,2$, and taking into account (4.1), we obtain

$$
\left\langle w_{1}, w_{2}\right\rangle=\left\langle d \psi_{p}\left(w_{1}\right), d \psi_{p}\left(w_{2}\right)\right\rangle=g_{M}\left(d\left(\pi_{M} \circ \psi\right)_{p}\left(w_{1}\right), d\left(\pi_{M} \circ \psi\right)_{p}\left(w_{2}\right)\right)
$$

and this completes the proof.

Remark 4.3. Observe that the map $\pi_{M} \circ \psi: \Sigma^{n} \rightarrow M^{n}$ in previous result is always open. If $\Sigma^{n}$ is compact, then $\pi_{M} \circ \psi$ is also closed. Therefore, in this case, $\pi_{M} \circ \psi$ is onto and $M^{n}$ must be also compact. Thus, Lemma 4.2 may be used to give an obstruction to the existence of codimension two compact spacelike submanifolds in a plane fronted wave spacetime $\bar{M}^{n+2}$ that factorizes through a characteristic hypersurface $u=u_{0}$

Remark 4.4. Observe that if $\Sigma^{n}$ factorizes through the characteristic hypersurface $u=u_{0}$, then the composition $\tilde{\psi}$ of $\psi$ with the isometry of a plane fronted wave spacetime $\bar{M}^{n+2}$ given by $(u, v, x) \mapsto$ $\left(u-u_{0}+u_{1}, v, x\right)$, satisfies that $\tilde{\psi}\left(\Sigma^{n}\right)$ lies in $u=u_{1}$. The extrinsic geometries of $\psi$ and $\tilde{\psi}$ are clearly the same. Thus, in order to study a codimension two spacelike submanifold of $\bar{M}^{n+2}$ through a characteristic hypersurface $u=u_{0}$, the value $u_{0}$ is completely irrelevant.

Remark 4.5. If in Lemma 4.2, the codimension of $\Sigma^{k}$ is allowed to be bigger than two, then $\pi_{M} \circ \psi$ is an immersion with $\left(\pi_{M} \circ \psi\right)^{*} g_{M}=\langle$,$\rangle on \Sigma^{k}$. 
Under the same assumptions and notation of Lemma 4.2, the next result is a consequence of [9, Lemma 7.3.3],

Proposition 4.6. If $\Sigma^{n}$ is complete, then $\pi_{M} \circ \psi: \Sigma^{n} \rightarrow M^{n}$ is a Riemannian covering. In particular, if $M^{n}$ is simply connected, then $\pi_{M} \circ \psi$ is a global isometry.

Let $\psi: \Sigma^{n} \rightarrow \bar{M}^{n+2}$ be a codimension two spacelike submanifold through a characteristic hypersurface $u=u_{0}$. In this case we have $\partial_{v}^{\perp}=\partial_{v}$ and $\partial_{v}^{\top}=0$ and then, equations (2.7) and 2.8) read, respectively, as follows

$$
A_{\partial_{v}}=0 \quad \text { and } \quad \nabla^{\perp} \partial_{v}=0 .
$$

Let us consider the normal vector field

$$
\eta=-\partial_{u}^{\perp}+\frac{1}{2}\left\langle\partial_{u}^{\perp}, \partial_{u}^{\perp}\right\rangle \partial_{v}
$$

that satisfies $\langle\eta, \eta\rangle=0$ and $\left\langle\eta, \partial_{v}\right\rangle=-1$. With respect to the global normal reference frame $\left\{\eta, \partial_{v}\right\}$ and taking into account (4.7), the second fundamental form of $\Sigma^{n}$ is given by

$$
\amalg(V, W)=-\left\langle A_{\eta} V, W\right\rangle \partial_{v}=\left\langle A_{\partial_{u}^{\perp}} V, W\right\rangle \partial_{v},
$$

for any $V, W \in \mathfrak{X}(\Sigma)$. Observe that the image of the second fundamental form is contained in the lightlike line spanned by $\partial_{v}$ at any point of $\Sigma^{n}$. Consequently, the mean curvature vector field is

$$
\mathbf{H}=\frac{1}{n} \operatorname{trace}\left(A_{\partial_{u}^{\perp}}\right) \partial_{v}
$$

and, therefore, we have that $\langle\mathbf{H}, \mathbf{H}\rangle=0$.

Now, decomposing $\partial_{u}$ into tangent and normal components and using (2.3) and (2.4), we get from (4.3)

$$
\nabla_{W} \partial_{u}^{\top}=A_{\partial_{u}^{\perp}}(W), \quad \nabla_{W}^{\perp} \partial_{u}^{\perp}=-\amalg\left(W, \partial_{u}^{\top}\right)+\frac{1}{2}\left\langle\nabla H_{u},\left(\pi_{M} \circ \psi\right)_{*}(W)\right\rangle \partial_{v} .
$$

Therefore, formulas (2.6) and (4.6) permit that 4.10) may be rewritten as

$$
\mathbf{H}=\frac{1}{n} \operatorname{div}\left(\partial_{u}^{\top}\right) \partial_{v}=\frac{1}{n} \Delta(v \circ \psi) \partial_{v} .
$$

In particular, in the case that $\mathbf{H}$ does not vanish everywhere on $\Sigma^{n}$, previous formula implies that $\Sigma^{n}$ is marginally trapped.

Remark 4.7. Taking into account (4.10), Corollary 2.6 may be sharpen in a special case,

Let $\psi: \Sigma^{n} \rightarrow \bar{M}^{n+2}$ be a compact codimension two spacelike submanifold in a plane fronted wave spacetime $\bar{M}^{n+2}$. Then, $\mathbf{H}=f \partial_{v}^{\perp}$, for some $f \in C^{\infty}\left(\Sigma^{n}\right)$, if and only if $\Sigma^{n}$ factorizes through a characteristic hypersurface $u=u_{0}$. 
Theorem 4.8. There exists no compact codimension two weakly trapped submanifold in a plane fronted wave spacetime $\bar{M}^{n+2}$.

Proof. Suppose the contrary to our claim. Then, from Remark 2.2, we know that $\Sigma^{n}$ factorizes through a certain characteristic hypersurface $u=u_{0}$. Using now equation (4.12) and the fact that $\mathbf{H}$ never vanishes on $\Sigma^{n}$, we obtain either $\Delta(v \circ \psi)<0$ or $\Delta(v \circ \psi)>0$ which is impossible because $\Sigma^{n}$ is assumed to be compact.

There is a distinguished family of codimension two spacelike submanifolds in a plane fronted wave spacetime $\bar{M}^{n+2}$ through the characteristic hypersurface $u=u_{0}$, namely, the spacelike graphs on an open domain $\Omega$ of $M^{n}$. They are defined as follows, given $h \in C^{\infty}(\Omega)$, and $u_{0} \in \mathbb{R}$, we put

$$
\sum_{u_{0}}^{n}(h):=\left\{\left(u_{0}, h(x), x\right) \in \mathbb{R} \times \mathbb{R} \times M^{n}: x \in \Omega\right\} .
$$

The graph $\Sigma_{u_{0}}^{n}(h)$ is a codimension two spacelike submanifold, for any $h$ and $u_{0}$, embedded by

$$
\psi_{h}: \Omega \rightarrow \bar{M}^{n+2}, \quad x \mapsto \psi_{h}(x):=\left(u_{0}, h(x), x\right),
$$

It should be pointed out that the induced metric $\psi_{h}^{*}\langle$,$\rangle on \Omega$ equals to $g_{\left.M\right|_{\Omega}}$ and, therefore, it does not depend on the function $h$.

Note that a spacelike immersion $\psi: M^{n} \rightarrow \bar{M}^{n+2}$ is a spacelike graph if and only if it factorizes through a characteristic hypersurface $u=u_{0}$ and $\pi_{M} \circ \psi$ is the identity map.

For every $V \in \mathfrak{X}(M)$, we have $d \psi_{h}(V)=V(h) \partial_{v}+V$. Then, it is easily checked that for each $x \in M^{n}$,

$$
T_{x}^{\perp} M=\operatorname{Span}\left\{\partial_{u}-\nabla h, \partial_{v}\right\} .
$$

As a direct consequence of 4.15, we get $\partial_{u}^{\top}=\nabla h$ and then 4.12 gives

$$
\mathbf{H}=\frac{\Delta h}{n} \partial_{v} .
$$

Equation (4.16) says that a graph $\psi_{h}: M^{n} \rightarrow \bar{M}^{n+2}$ in a plane fronted wave spacetime through a characteristic hypersurface $u=u_{0}$ satisfies $\mathbf{H}=r \partial_{v}$, for some $r \in \mathbb{R}$, if and only if $\Delta h$ is constant.

A spacelike submanifold $\psi: \Sigma^{n} \rightarrow \bar{M}^{n+2}$ in a plane fronted wave spacetime $\bar{M}^{n+2}$ is called a front of wave [11] if

$$
u \circ \psi=u_{0} \quad \text { and } \quad v \circ \psi=v_{0},
$$

for real constants $u_{0}, v_{0}$. Note that embedded front of waves provide a foliation by codimension two totally geodesic spacelike submanifolds of the plane fronted wave spacetime. As an immediate application of (4.16), we get,

The only compact entire spacelike graphs $\sum_{u_{0}}^{n}(h)$ that satisfy $\mathbf{H}=0$ are the front of waves $h=v_{0}, u=u_{0}$. 
Remark 4.9. Clearly, from 4.7) previous statement also works if the assumption $\mathbf{H}=0$ is changed to the more general one $\nabla_{V}^{\perp} \mathbf{H}=0$ for all $V \in \mathfrak{X}\left(\Sigma_{u_{0}}^{n}(h)\right)$, where $\nabla^{\perp}$ is the normal connection (compare with [4]).

For any front of wave $\psi: \Sigma^{n} \rightarrow \bar{M}^{n+2}$ in a plane fronted wave spacetime $\bar{M}^{n+2}$, we have that $\left\{\partial_{u}, \partial_{v}\right\}$ is a basis of $\mathfrak{X}^{\perp}(\Sigma)$ and satisfies

$$
\left\langle\partial_{u}, \partial_{u}\right\rangle=H, \quad\left\langle\partial_{v}, \partial_{v}\right\rangle=0, \quad \text { and } \quad\left\langle\partial_{u}, \partial_{v}\right\rangle=1
$$

Hence, we have $\partial_{u}=\partial_{u}^{\perp}$ and equation 4.3 gives rise the Weingarten formula for $\partial_{u}^{\perp}$

$$
\bar{\nabla}_{W} \partial_{u}^{\perp}=\frac{1}{2} g_{M}\left(\widetilde{\nabla} H_{u},\left(\pi_{M} \circ \psi\right)_{*}(W)\right) \partial_{v}^{\perp},
$$

where $W \in \mathfrak{X}(\Sigma)$. In particular, $A_{\partial_{u}}$ vanishes identically and therefore, every front of wave is totally geodesic.

Theorem 4.10. Let $\psi: \Sigma^{n} \rightarrow \bar{M}^{n+2}$ be a codimension two spacelike submanifold in a plane fronted wave spacetime $\bar{M}^{n+2}$ with signed $\left\langle\mathbf{H}, \partial_{v}\right\rangle$. Assume $\Sigma^{n}$ is compact. Then, the following assertions are satisfied

i) $\Sigma^{n}$ factorizes through a characteristic hypersurface $u=u_{0}$.

ii) $\sum^{n}$ is a Riemannian covering space of $M^{n}$, in particular, $M^{n}$ is also compact.

If in addition $\mathbf{H}=0$, then $\psi$ is a front of wave and, in particular, it is totally geodesic.

Proof. Formula (4.5) shows that $u \circ \psi$ must be a constant $u_{0}$. Hence, $\Sigma^{n}$ factorizes through a characteristic hypersurface $u=u_{0}$, which proves $i$ ). On the other hand, from Lemma 4.2 we know that $\Sigma^{n}$ is locally isometric to $M^{n}$. Actually, since $\Sigma^{n}$ is compact, Proposition 4.6 states that $\Sigma^{n}$ is a covering space of $M^{n}$, in particular, $M^{n}$ is compact, proving $i$ ). Finally $\mathbf{H}=0$ and (4.12) imply $v \circ \psi$ is also constant.

Remark 4.11. If the assumption $\mathbf{H}=0$ is dropped in previous result, then the conclusion is not achieved. In fact, consider $\Sigma^{n}=M^{n}=\mathbb{S}^{n}$ the unit $n$-dimensional sphere with its canonical metric. Let $h \in C^{\infty}\left(\mathbb{S}^{n}\right)$ be a non-constant function satisfying $\Delta h+n h=0$, i.e., an eigenfunction corresponding to the first non-trivial eigenvalue $\lambda_{1}=n$ of $\mathbb{S}^{n}$. Then, the graph on $\mathbb{S}^{n}$ in a plane fronted wave spacetime $\bar{M}^{n+2}$ through the characteristic hypersurface $u=u_{0}$ defined by $h$ satisfies $\mathbf{H}=-h \partial_{v}$ from 4.16 . Hence, $\left\langle\mathbf{H}, \partial_{v}\right\rangle=0$ and clearly $\Sigma^{n}$ is not totally geodesic.

Proposition 4.12. Let $\psi: \Sigma^{n} \rightarrow \bar{M}^{n+2}$ be a codimension two spacelike submanifold which factorizes through a characteristic hypersurface $u=u_{0}$. If $\bar{M}^{n+2}$ satisfies the Timelike Convergence Condition (TCC) then, the Ricci tensor of $\Sigma^{n}$ is positive semi-definite. 
Proof. Recall that, from Lemma 4.2, $\Sigma^{n}$ is locally isometric to $M^{n}$, and hence, the Ricci tensor of $\Sigma^{n}$ satisfies Ric $=\left(\pi_{M} \circ \psi\right){ }^{*} \operatorname{Ric}_{M}$. Moreover, since $\bar{M}^{n+2}$ satisfies the TCC, from (4.4) we have that Ric $_{M}$ is positive semi-definite, and therefore Ric is also positive semi-definite, ending the proof.

Theorem 4.13. Let $\psi: \Sigma^{n} \rightarrow \bar{M}^{n+2}$ be a complete codimension two spacelike submanifold with $\mathbf{H}=0$ which factorizes through a characteristic hypersurface $u=u_{0}$. If $\bar{M}^{n+2}$ satisfies the TCC and the function $v \circ \psi$ is bounded from above or from below, then $\Sigma^{n}$ is a front of wave and, in particular, it is totally geodesic.

Proof. By Proposition 4.12 we know that the Ricci tensor of $\Sigma^{n}$ is positive semi-definite. Moreover, taking into account the expression obtained in (4.12), if $\Sigma^{n}$ is stationary then $\Delta(v \circ \psi)=0$. Since we are assuming $v \circ \psi$ to be bounded, by [26, Corollary 1], $v \circ \psi$ is a constant function, and hence, $\Sigma^{n}$ is a front of wave.

\section{Conclusion}

We have studied along this paper spacelike submanifolds with codimension $\geq 2$ in a Brinkmann spacetime. The symmetry of such a spacetime defined by a lightlike parallel vector field $\xi$ plays a crucial role in our approach. Indeed, we have found sufficient conditions in terms of the mean curvature vector field so that a spacelike submanifold is included in a leaf of the foliation tangent vectors orthogonal to $\xi$. Each leaf of this foliation is called a characteristic hypersurface. The geometry of such a hypersurface is degenerate. Therefore, if a spacelike submanifold in a Brinkmann spacetime is contained in a characteristic hypersurface, this reduction of codimension is not so significant as in the case of a submanifold in a Riemannian manifold. In particular, the extrinsic geometry of a spacelike hypersurface in a leaf is completely different to the one in the case of a hypersurface in a Riemannian manifold or a spacelike hypersurface in a spacetime.

Then, we have considered plane fronted wave spacetimes, a particular case of Brinkmann spacetimes, that naturally generalize classical pp-waves spacetimes. A plane fronted wave spacetime is foliated by codimension two totally geodesic spacelike submanifolds, each of them called a front of wave. Each front of wave is contained in a characteristic hypersurface. Thus, the idea to characterize globally front of waves naturally arises. In this direction we have first studied under that assumptions a compact codimension two spacelike submanifold in a plane fronted wave spacetime is contained in a characteristic hypersurface and later, several uniqueness results for front of waves. We have proven specifically that any compact codimension two spacelike submanifold with zero mean curvature vector field in a plane fronted spacetime wave must be a front of wave (totally geodesic in particular).

Acknowledgements. The authors would like to give their sincere thanks to the referees for the careful reading of the manuscript and their corresponding suggestions. 


\section{References}

[1] B.P. Abbott et al., Observation of gravitational waves from a binary black hole merger, Phys. Rev. Lett. 116 (2016), 1-16.

[2] L.J. Alías, V.L. Cánovas and M.Rigoli, Trapped submanifolds contained into a null hypersurface of de Sitter spacetime, Commun. Contemp. Math. 20 (2018), 1750059 (30 pp.).

[3] L.J. Alías, V.L. Cánovas and M.Rigoli, Codimension two spacelike submanifolds into a null hypersurface of the Lorentz-Minkowski space. Proc. Roy. Soc. Edinburgh 149A (2019), 15231553.

[4] L.J. Alías, F.J. Estudillo and A. Romero, Spacelike submanifolds with parallel mean curvature in pseudo-Riemannian space forms, Tsukuba J. Math. 21 (1997), 169-179.

[5] J.L. Alías, A. Romero, M. Sánchez, Uniqueness of complete spacelike hypersurfaces of constant mean curvature in generalized Robertson-Walker spacetimes, Gen. Rel. Grav. 27 (1995), 71-84.

[6] J.K. Beem, P.E. Ehrlich and K.L. Easley, Global Lorentzian geometry, Monographs Textbooks Pure Appl. Math. 202, Dekker Inc., New York, 1996.

[7] H.W. Brinkmann, Einstein spaces which are mapped conformally on each other, Math. Ann. 94 (1925), 119-145.

[8] A. Candela, J.L. Flores and M. Sánchez, On general plane fronted waves. Geodesics, Gen. Relativity Gravit. 4 (2003), 631-649.

[9] M.P. do Carmo, Riemannian Geometry, Birkhäuser, Boston, Basel, Berlin, 1992.

[10] S.T. Cheng, S.T. Yau, Maximal spacelike hypersurfaces in the Lorentz-Minkowski space, Ann. of Math. 104 (1976), 407-419.

[11] J.L. Flores and M. Sánchez, On the geometry of pp-wave type spacetimes, Analytical and numerical approaches to mathematical relativity, Lecture Notes in Phys., 692, Springer, Berlin, (2006) 79-98.

[12] J.L. Kazdan, Parabolicity and the Liouville property on complete Riemannian manifolds, Aspects of Math. E10, Ed. A.J. Tromba, Friedr. Vieweg and Sohn, Bonn (1987), 153-166.

[13] M. Mars and J.M.M. Senovilla, Trapped surfaces and symmetries, Class. Quantum Grav., 20 (2003), 293-300.

[14] B. O’Neill, Semi-Riemannian Geometry with applications to Relativiy, Pure and applied Math 103, Academic Press 1983. 
[15] F.J. Palomo and A. Romero, On spacelike surfaces in four-dimensional Lorentz-Minkowski spacetime through a light cone, Proc. Royal Soc. Edinburgh 143A (2013), 881-892.

[16] J.A.S. Pelegrín, A. Romero and R.M. Rubio, On maximal hypersurfaces in Lorentz manifolds admitting a parallel lightlike vector field, Class. Quantum Grav., 33 (2016), 055003(8 pp.).

[17] R. Penrose, Gravitational collapse and Space-time Singularities, Physical Review Letters 14 (1965), 57-59.

[18] R. Penrose, Any space-time has plane wave as a limit, Differential Geometry and Relativity, A volume in honour of André Lichnerowicz on his 60th birthday, M. Cahen, M. Flato (eds.), 3 (1976), 271-275.

[19] A. Romero, Constant mean curvature spacelike hypersurfaces in spacetimes with certain causal symmetries, Hermitian-Grassmannian Submanifolds, Daegu (Korea), Y.J. Suh et al. (eds.), Springer Proceedings in Mathematics and Statistics 203 (2017), 1-15.

[20] A. Romero, R.M. Rubio and J.J. Salamanca, Uniqueness of complete maximal hypersurfaces in spatially parabolic generalized Robertson-Walker spacetimes, Class. Quantum Grav., 30 (2013), 115007(13 pp.).

[21] R.K Sachs and H. Wu, General Relativity for Mathematicians, Grad. Text. in Math., 48, Springer-Verlag, 1977.

[22] H. Stephani, D. Kramer, D. Maccallum, C. Hoenseloers and E. Herlt, Exact solutions to Einstein's field equations (2th edition), Cambridge University Press, Cambridge, U.K. 2003.

[23] M.A.L. Velásquez, H.F. de Lima, Complete spacelike hypersurfaces immersed in pp-wave spacetimes Gen. Relativity Gravit., 52 (2020), article number 41, 1-18.

[24] A. G. Walker, On parallel fields of partially null vector spaces, Quart. J. Math. 20 (1949), 135145.

[25] H-H. Wu, The Bochner Technique in Differential Geometry, Classical Topics in Mathematics, 6, Higher Education Press, Beijing, 2017.

[26] S.T. Yau, Harmonic functions on complete Riemannian manifolds, Comm. Pure and Applied Math. 28 (1975), 201-228.

[27] S.T. Yau, Some function-theoretic properties of complete Riemannian manifolds and their applications to geometry, Indiana Univ. Math. J. 25 (1976), 659-670. 